\title{
Article@Virology
}

\section{Cross-Neutralizing Activity of Monoclonal Antibodies Against N501Y Mutant Strain of SARS-CoV-2}

Ruxia Ding ${ }^{l} \dagger$, Haixin Wang ${ }^{1,2} \dagger$, Yi Yang ${ }^{3}$, Liangshu Xie ${ }^{3}$, Li Zhang ${ }^{1}$, Qianqian Li ${ }^{1}$, Shuo Liu ${ }^{1}$, Jianhui Nie ${ }^{1}$, Jiajing Wu ${ }^{1}$,Haiyang Qin $^{1}$, Yuelei Shen ${ }^{3 *}$, Huiguo Wang ${ }^{2 *}$, Weijin Huang ${ }^{1 *}$ and Youchun Wang ${ }^{l *}$

1. Division of HIV/AIDS and Sex-transmitted Virus Vaccines, Institute for Biological Product Control, National Institutes for Food and Drug Control and WHO Collaborating Center for Standardization and Evaluation of Biologicals, Beijing, 102629, P.R. China.

2. Department of pharmaceutical engineering, college of life science and technology, Dalian University,

Dalian, 116622,P.R. China.

3. Beijing Biocytogen Co., LTD , Beijing 102629,P.R. China

\begin{abstract}
The dominant N501Y mutation in the spike protein that SARS-CoV-2 virus uses to bind to the human ACE2 receptor were found in the UK, which has aroused global concern and worried. Mutations in spike protein may, in theory, result in more infectious and spreading more easily. In order to evaluate the broad-spectrum protective effect of the monoclonal antibodies(mAbs), we compared the neutralization activities of six prepared mAbs against SARS-CoV-2 with pseudovirus neutralization assay. Only one of them showed a decrease of 6 folds in neutralizing activity to N501Y mutant strain, compared with the wild type strain. We should continue to monitor emergence of new variants in different regions to study their infectivity and neutralization effect.

Copyright@2012-2025 Published by Hong Kong Institute of Biologicals Standardization Limited. All rights reserved.
\end{abstract}

Article history: Submitted: 18/12/2020; Revised: 24/12/2020; Accepted: 25/12/2020

DOI: $10.21092 /$ jav.v9i4.90

Key Words: COVID-19, SARS-CoV-2, Mutation, Antibody

$\dagger$ These authors contributed equally to this work

* Corresponding author, requests for materials should be addressed to Prof. Huang (huangweijin@nifdc.org.cn), or Prof.Wang (wangyc@nifdc.org.cn)

Copyright@2012-2020 Published by Hongkong Institute of Biologicals Standardization Limited. All rights reserved. 
Anti-viral monoclonal antibodies(mAbs) have been successfully used in the clinical treatment of respiratory syncytial virus (RSV) and Ebola virus (EVD) ${ }^{[1]}$. For the current pandemic of the COVID-19, some mAbs for SARS-CoV-2 have been in the pipeline of clinical trial. We expect to get mAbs with cross-neutralizing protective effect against the wild type strain and mutant strains of SARS-CoV-2. Here, we obtained 6 kinds of mAbs with potentially blocking effect through separation and purification from the serum of mice immunized with recombinant $\mathrm{S}$ protein. The SARS-CoV-2 pseudovirus was used to evaluate their neutralizing and protective effects on wild strain and the N501Y mutant strain.

\section{Materials and Method}

\section{Pseudovirus Neutralization assay}

The N501Y mutant pseudovirus was constructed on the basis of wild type strain, described by $\mathrm{Li}$ et $\mathrm{al}^{[2]}$. Add $100 \mu \mathrm{L}$ of the serial dilution of the $\mathrm{mAb}$ sample to $\mathrm{a}$ 96-well plate, and then add $50 \mu \mathrm{L}$ of pseudoviruses with a concentration of $1300 \mathrm{TCID}_{50} / \mathrm{ml}$ and incubate at $37^{\circ} \mathrm{C}$ for $1 \mathrm{~h}$. After that, $100 \mu \mathrm{L}$ of Huh-7 cells (2000/well) were added to the plate, and incubated in a $37^{\circ} \mathrm{C}, 5 \% \quad \mathrm{CO}_{2}$ incubator. After $24 \mathrm{~h}$, chemiluminescence detection was performed, and the virus neutralization titer was calculated using the Reed-Muench method.

2. Monoclonal antibodies against SARS-CoV-2

6 kinds of mAbs against SARS-CoV-2 were provided by Biocytogen Ltd.
The pathogen-free 6-8weeks female mice were immunized with $20 \mu \mathrm{g}$ purified recombinant $\mathrm{RBD}$ protein of the SARS-CoV-2. The spleens of mice were fused with SP2/0 cell line and the cells were transferred to a 96-well plate and cultured at $37^{\circ} \mathrm{C}$ in the environment of $8 \% \mathrm{CO}_{2}$. The positive clones of hybridoma fusion were sreeened by blocking RBD binding to human ACE2.

The RNA was extracted from hybridoma cells and reverse transcriped into cDNA. The target fragments obtained by PCR augment were linked into the vector of pEE12.4 in order to harvest the plasmids containing of the fully human antibody light and heavy chains. The subtype of antibodies were $\operatorname{IgG}_{1}$. These plasmids were transiently transferred into $\mathrm{CHO}-\mathrm{S}$ cells and the purified antibodies were obtained by AKTA. The binding of the antibody to the RBD was detected using a Biacore $8 \mathrm{~K}$. The antibodies showed superior affinity to the RBD protein.

\section{Results and Conclusion}

As an RNA virus, SARS-CoV-2 is tends to mutate in the process of transmission, we noticed that the N501Y mutant strain has grown rapidly in recent months, up to now, virus strains containing N501Y mutation have reached $1.4 \%$ of the total number of mutants. The growth trend of N501Y mutant strains in different countries was shown in Figure 1. This mutation first appeared in Brazil in April. In June, Mutant strains at this site appeared in Australia and the United 
Kingdom. Beginning in September, the no neutralizing activity to both strains. mutant strain began to surge in the UK. Among the other four mAbs, the N501Y Among the newly added sequences mutation reduced the neutralization containing the N501Y mutation in December, sensitivity with mAb 03-1F9 by 6 folds, $93.7 \%$ of the sequences appeared in the UK. compared with that of wild strain. EC50 of At the same time we noticed that the N501 site is located at the key position where Spike protein binds to ACE2, and its $\mathrm{mAb} 03-1 \mathrm{~F} 9$ decreased from $0.15 \mathrm{ng} / \mathrm{ml}$ to $0.97 \mathrm{ng} / \mathrm{ml}$. No significant differeance to the mutation may affect its binding to antibodies, and even affect the protective effect of the vaccine. It has been reported that mutations at this site greatly enhance the binding affinity of the virus to the receptor, This requires us to always pay attention to the changes in infectivity and neutralizing activity caused by mutations.

In this study, we evaluated the neutralization titers of $6 \mathrm{mAbs}$ against the wild strain of SARS-CoV-2 and the N501Y mutant strain. Among them, two mAbs have sensitivity of mAbs 09-7B8, 09-4E5-1G2, and 09-2F7-1A1 was found (Details see figure 2).

Since the outbreak of the SARS-CoV-2, great efforts were put in all over the world looking for prevention and treatment of this disease. The effect of mAbs, which block the interaction between SARS-CoV-2 RBD and the receptor $\mathrm{ACE} 2$, is one of the effective ways to deal with SARS-CoV-2 infection ${ }^{[3]}$. We used the SARS-CoV-2 pseudovirus system to evaluate the infectivity of 106 strains of mutations and the neutralizing

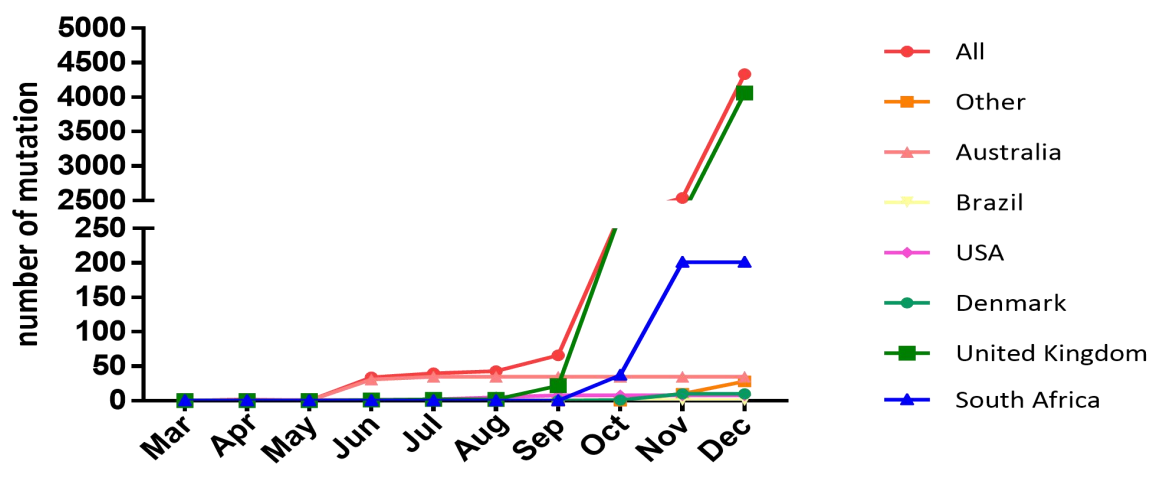

Figure 1. The growth trend of N501Y mutant strains.

Statistics of the growth of N501Y mutant strains in different countries from March to December. 


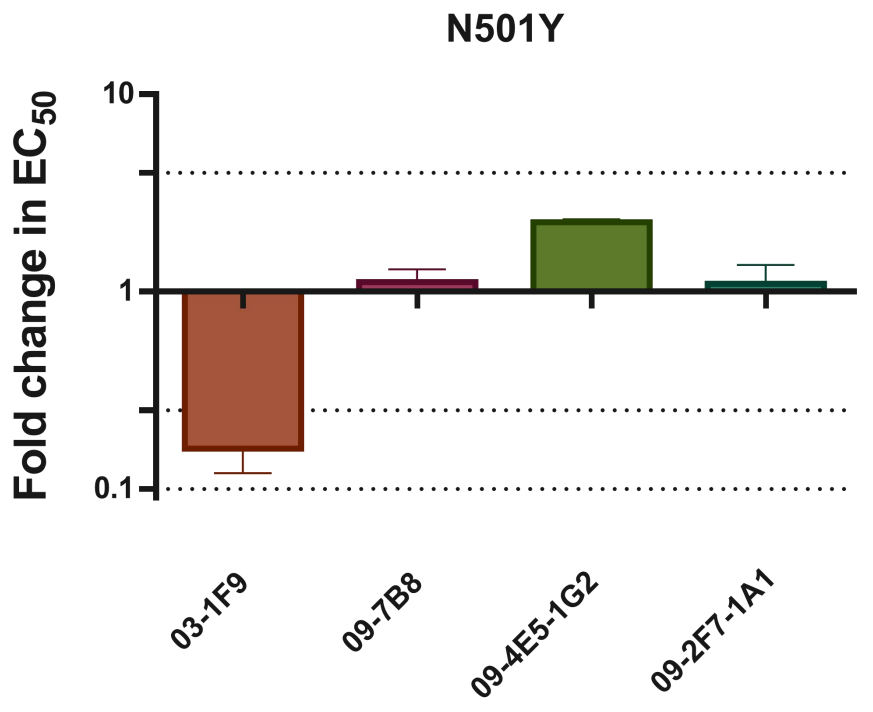

Figure 2. Analysis of neutralization and protection effect of mAbs on wild strains and N501Y mutant strains.

The ratio of EC50 of the mAb against the wild strain(EC50-WT) to the EC50 of mAbs against the N501Y(EC50-501Y) was calculated(EC50-WT/EC50-501Y). The data is a result of three repetitions.

activity of mAbs, but new mutations continue to emerge, and some new mutations continue to attract everyone's attention, worrying that their increased infectivity will increase the transmission ability, or lead to the escape of existing vaccines and antibodies. Pseudovirus technology can be used to evaluate the best broad-spectrum protection method for different mutant strains. Using this technique, we evaluated the mAbs neutralizing activity of the N501Y mutant strain, which is currently surging rapidly in the UK, firstly N501Y mutant pseudovirus was constructed, and used to evaluate the neutralization activity of the above $4 \mathrm{mAbs}$, and it was found that the mutation at this site lead to neutralization activity to mAb 03-1F9 decreased. In other studies, It was also found that mutations in the RBD site caused the escape of mAbs, mutations increase the infectivity of the virus in a mouse infection model ${ }^{[2,4]}$,This suggests that mutations cause the risk of antibody escape and increased infectivity. We should closely track the site mutations to find a $\mathrm{mAb}$ with high protection. If a single antibody cannot achieve comprehensive protection, studies have shown that the combined application of multiple antibodies, namely cocktail therapy, has a better protective effect on the SARS-CoV-2 ${ }^{[5]}$. At the same time, we should continue to monitor the emergence of new variants in different regions to study their infectivity 
and neutralization effect. There is no doubt that the pseudovirus technology provides a convenient and fast method.

\section{Acknowledgments}

This work was supported by General Program of National Natural Science Foundation of China (Grant number 82073621), and the Bill \& Melinda Gates Foundation (Investment ID INV-006379).

\section{Reference}

[1]. Tuccori M, Ferraro S, Convertino I, et al. Anti-SARS-CoV-2 neutralizing monoclonal antibodies: clinical pipeline[C]//mAbs. Taylor \& Francis, 2020, 12(1): 1854149.
[2]. Li Q, Wu J, Nie J, et al. The impact of mutations in SARS-CoV-2 spike on viral infectivity and antigenicity[J]. Cell, 2020, 182(5): 1284-1294. e9..

[3]. Sajna K, Kamat S. Antibodies at work in the time of severe acute respiratory syndrome coronavirus 2[J]. Cytotherapy, 2020.

[4]. Gu H, Chen Q, Yang G, et al. Adaptation of SARS-CoV-2 in BALB/c mice for testing vaccine efficacy[J]. Science, 2020, 369(6511): 1603-1607.

[5]. Baum A, Fulton B O, Wloga E, et al. Antibody cocktail to SARS-CoV-2 spike protein prevents rapid mutational escape seen with individual antibodies[J]. Science, 2020, 369(6506): 1014-1018. 